Publ. RIMS, Kyoto Univ.

12 Suppl. (1977), 347-349.

\title{
Progress in Analytic S-Matrix Theory
}

\author{
by \\ David OlIVE*
}

First I should like to say how honoured and happy I feel to be here in Japan attending the OJI seminar on algebraic analysis organised by Professor Sato. Thank you.

The reason I am here is that hyperfunctions provide an ideal mathematical framework for analytic $S$-matrix theory. Since it is now 9 years since I stopped working on the analytic $S$-matrix I shall ask you to excuse me for giving a talk which will make rather general points and not be technical. I shall concern myself with the progress in analytic $S$-matrix theory and pose some questions.

Right at the beginning Heisenberg realised the importance of both the many particle structure of the $S$-matrix and its analyticity. The importance of these twin themes was borne upon me in 1963 when I considered the problem of how to set up an axiomatic framework for $S$-matrix theory. At that time the triumphs of axiomatic quantum field theory were the deductions of certain general theorems such as the TCP theorem and the connection between spin and statistics from a few rather general principles. Why could not the same be done for the $S$-matrix? We would then learn what were the relevant underlying principles and structure. Properties like "crossing" and "hermitian analyticity" which were so essential to the calculations of the day referred to analytic continuations from one physical region to another. The physical region, and its immediate complex neighbourhood, is the region in which the $S$-matrix is measured (since it corresponds to the participating particles being real and physical). Properties involving analyticity well away from these regions should surely not be postulated but rather deduced from more

Received October 12, 1976.

* CERN, Geneva and Niels Bohr Institute, Copenhagen. 
fundamental principles involving the physical region.

Such was my belief. The insight that encouraged this belief that the physical regions could indeed control the global properties met above was the realisation of the enormous amount of structure which manifests itself in the physical regions of the many particle $S$-matrix elements.

Arguments were made to illustrate the power of this structure: crossing, hermitian analyticity, the TCP theorem (and later the connection between spin and statistics) were made extremely plausible. To strengthen the arguments it became necessary to understand the physical regions better. After 13 years we are still working at this, and it has become an extremely interesting pursuit in its own right. Although the general features of the physical region were appreciated a detailed, precise and comprehensive mathematical treatment was lacking. The concepts of Landau singularities and their associated $i \epsilon$ prescription were formulated and it was understood that this structure was controlled by the unitarity equations. The problem was (and still is) to find the precise degree of control. In the early studies the relevant mathematics was made up ad hoc by physicists.

At the Nice Symposium in 1973 we learnt how the precise mathematical concept of "microanalyticity" fits in with $S$-matrix ideas. In particular it provided a more rigorous and general formulation of the old concept of if prescription and fitted very naturally with the physical idea of macrocausality. I discussed my point of view on this at Nice and shall not repeat myself now.

Here in Kyoto we have learnt of some new ideas: that Landau varieties constitute "holonomic varieties". Nowadays the Landau equations are understood to involve momentum space " $p$ " (as usual) but also co-ordinate space-time " $x$ " which is now used to formulate the modern version of the $i \epsilon$ prescription. A variety $L(x, p)=0$ is holonomic if (i) it is defined by as many independent equations as there are variables $p$, and if (ii) the Poisson bracket with respect to $x, p$ of any two functions vanishing on $L=0$ itself vanishes.

Inspired by this result Sato has proceeded a step further to make an intriguing conjecture: the $S$-matrix (at least in the physical region) 
solves a holonomic system of linear micro-differential equations, i.e. the micro-differential equations are self consistent and there are as many equations as momentum variables. Then the solutions can only be singular on a set of holonomic varieties called the characteristic varieties of the equations, of course the Landau varieties. The solutions, called holonomic functions have certain specific properties; e.g. $s^{t}$ is not a holonomic function of $s$ and $t$, and hence the conjecture of Sato is nontrivial in content.

A first step towards verifying the conjecture has been to check it for any Feynman integral. I have been impressed by the progress made by the young men of Kyoto.

Now I want to ask some questions. The fundamental equations of $S$-matrix theory are the unitarity equations expressing the fact that since $S$ is a probability amplitude for a scattering process the corresponding probabilities must sum to one. This, combined with the connectedness structure, is the input for the particle structure which then manifests itself via the Landau varieties. The unitarity equations are in effect complicated non-linear equations whereas Sato's equation is linear, and hence easier to analyse. What I want to know is how much of the structure implied by unitarity can be stored in Sato's equation and then reproduced by its solution?

More specifically:-

1) Does unitarity determine Sato's equations? If so, how? (Kawai has argued that this follows from the discontinuity formulae which in turn are thought to follow from unitarity. Is there a more direct and precise route of construction?)

2) Is every solution to Sato's equations unitary? (Probably the answer is no: then we wonder how to select the unitary solutions)

3) What is the arbitrariness in unitary solutions?

4) How can we fix this arbitrariness?

5) What are the properties of the solution so found? Is it crossing symmetric etc. etc? (It should be if the old ideas are correct). 
